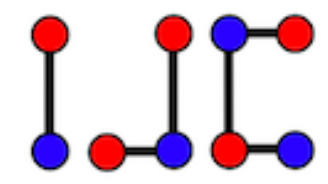

\title{
Super local edge anti-magic total coloring of paths and its derivation
}

\author{
Fawwaz Fakhrurrozi Hadiputra $^{\mathrm{a}}$, Denny Riama Silaban*a ${ }^{*}$ Tita Khalis Maryati ${ }^{\mathrm{b}}$ \\ ${ }^{a}$ Department of Mathematics, FMIPA, Universitas Indonesia, Depok, Indonesia \\ ${ }^{b}$ Department of Mathematics Education, FITK, UIN Syarif Hidayatullah, Ciputat, Indonesia \\ fawwazfh@sci.ui.ac.id, denny@sci.ui.ac.id, tita.khalis@uinjkt.ac.id
}

\begin{abstract}
Suppose $G(V, E)$ be a graph and suppose $u, v, x$ be vertices of graph $G$. A bijection $f: V \cup$ $E \rightarrow\{1,2,3, \ldots,|V(G)|+|E(G)|\}$ is called super local edge antimagic total coloring if for any adjacent edges $u v$ and $v x$ in $E(G), w(u v) \neq w(v x)$, which $w(u v)=f(u)+f(u v)+f(v)$ for $f(V)=\{1,2,3, \ldots,|V(G)|\}$. By giving $G$ a labeling $f$, we denotes the minimum number of distinct weight of edges needed in $G$ as $\gamma_{\text {sleat }}(G)$. In this study, we proved the $\gamma_{\text {sleat }}$ of paths and its derivation.
\end{abstract}

Keywords: Edge chromatic number, path graphs, super local edge antimagic, total coloring Mathematics Subject Classification: 05C05, 05C15, 05C38, 05C78

DOI: $10.19184 /$ ijc.2019.3.2.6

\section{Introduction}

For a graph $G$, a bijection $f: V \cup E \rightarrow\{1,2,3, \ldots,|V(G)|+|E(G)|\}$ assigns each edges to a particular distinct number from 1 up to $|V(G)|+|E(G)|$. Suppose $u, v, x$ be vertices of graph $G$. Weight of an edge $w(u v)$ is defined as $w(u v)=f(u)+f(u v)+f(v)$ for every edge $u v$ in $G$. A bijection $f$ is called local edge antimagic total coloring if any adjacent edges $u v$ and $v x, w(u v) \neq w(v x)$. For every distinct weight, we denote them as distinct colors. The local edge antimagic total chromatic number of $G, \gamma_{\text {leat }}(G)$, is the minimum number of colors for edges taken over all colorings induced by local antimagic total labelings of $G$. If vertices of $G$ are assigned smaller labels, then we call $f$ as super local edge antimagic total coloring of $G$, and the minimum number of colors as $\gamma_{\text {sleat }}$. For convenience, we will use the abbreviation SLEAT as super local

Received: 9 Jul 2019, Revised: 16 Oct 2019, Accepted: 15 Nov 2019.

*Corresponding Author: denny@sci.ui.ac.id (Denny Riama Silaban). 
edge anti-magic total. A SLEAT colorable graph is a graph that admits SLEAT coloring. Let $\gamma(G)$ be the edge chromatic number of $G$. We can observe that $\Delta(G) \leq \gamma(G)$, where $\Delta(G)$ is a maximum degree in graph $G$. A compilation of observation concludes that $\Delta(G) \leq \gamma(G) \leq$ $\gamma_{\text {leat }}(G) \leq \gamma_{\text {sleat }}(G)$ for an arbitrary graph $G$.

Ringel firstly introduced the term of antimagic labelings [6]. There are many researchs conducted in antimagic labelings and its variation based on the survey of Gallian [5]. One of many variations is local antimagic labelings.

Arumugam et al. introduced local antimagic as vertex local antimagic edge labelings [3]. Followed by labels addition for the vertex, vertex local antimagic total labelings, one described in by Kurniawati et al. [8]. The analog rises, edge local antimagic total labelings, explained by Agustin et al. in [1] that includes path graph. If every vertex labels are smaller than edge labels, then it is a super local edge antimagic total labelings, which Agustin et al. [2] and Kurniawati et al. [7] describe in some other graph.

In this paper, we study super local edge anti-magic total coloring of path graph, path with edge(s) addition which forms unicycle, connecting two disjoint path, and amalgamation of star. If $\gamma(G)=\gamma_{\text {sleat }}(G)$, then by the prior inequality, $\gamma_{\text {leat }}(G)=\gamma_{\text {sleat }}(G)$. In other words, studies about local edge anti-magic total (LEAT) coloring in all graphs that satisfy $\gamma(G)=\gamma_{\text {sleat }}(G)$ are not necessary.

\section{Main Results}

\subsection{Path Graphs}

First, we are going to establish SLEAT for paths. This theorem is used in every proceeding theorems.

Theorem 2.1. Let $n \geq 2$ be integer and $P_{n}$ be a path with $n$ vertices. $\gamma_{\text {sleat }}\left(P_{n}\right)=2$.

Proof. Let $V\left(P_{n}\right)=\left\{v_{i} \mid 1 \leq i \leq n\right\}$ and $E\left(P_{n}\right)=\left\{v_{i} v_{i+1} \mid 1 \leq i \leq n-1\right\}$. Since $\Delta\left(P_{n}\right)=2$, $\gamma_{\text {sleat }}\left(P_{n}\right) \geq 2$. To show $\gamma_{\text {sleat }}\left(P_{n}\right) \leq 2$, define $f: V\left(P_{n}\right) \cup E\left(P_{n}\right) \rightarrow\left\{1,2, \ldots,\left|V\left(P_{n}\right)\right|+\left|E\left(P_{n}\right)\right|\right\}$.

Case 1. $n$ is odd.

Labels vertices and edges as follows

$$
\begin{gathered}
f\left(v_{i}\right)=\left\{\begin{aligned}
\frac{i}{2}, & \text { if } i \text { is even, } \\
\frac{n+i}{2}, & \text { if } i \text { is odd, }
\end{aligned}\right. \\
f\left(v_{i} v_{i+1}\right)= \begin{cases}2 n-i+1, & \text { if } i \text { is even, } \\
2 n-i-1, & \text { if } i \text { is odd. }\end{cases}
\end{gathered}
$$

Hence, we get

$$
w\left(v_{i} v_{i+1}\right)=f\left(v_{i}\right)+f\left(v_{i} v_{i+1}\right)+f\left(v_{i+1}\right)= \begin{cases}\frac{5 n+1}{2}+1, & \text { if } i \text { is even } \\ \frac{5 n+1}{2}-1, & \text { if } i \text { is odd }\end{cases}
$$


Case 2. $n$ is even.

Labels vertices and edges as follows

$$
\begin{gathered}
f\left(v_{i}\right)=\left\{\begin{aligned}
n, & \text { if } i=1, \\
\frac{n+i}{2}-1, & \text { if } i \text { is even, } \\
\frac{i-1}{2}, & \text { if } \text { odd } i>1, \\
n+1, & \text { if } i=1, \\
2 n-i, & \text { if } i \text { is even, } \\
2 n-i+2, & \text { if odd } i>1 .
\end{aligned}\right.
\end{gathered}
$$

Hence, we get

$$
w\left(v_{i} v_{i+1}\right)=f\left(v_{i}\right)+f\left(v_{i} v_{i+1}\right)+f\left(v_{i+1}\right)= \begin{cases}\frac{5 n}{2}-1, & \text { if } i \text { is even } \\ \frac{5 n}{2}+1, & \text { if } i \text { is odd }\end{cases}
$$

Since in every case there is only two distinct $w$, therefore $f$ is a SLEAT labeling of $P_{n}$ with $\gamma_{\text {sleat }}\left(P_{n}\right)=2$.
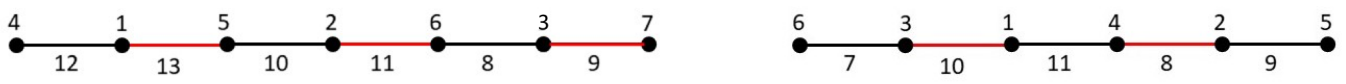

Figure 1: SLEAT coloring of $P_{7}$ and $P_{6}$

\subsection{Unicycle graph}

\subsubsection{Path with an edge addition}

Now, we start to add variation by adding an edge for path, forming a unicycle graph. We want to exclude the possibility of forming a regular cycle.

Theorem 2.2. Let $n \geq 3$ be integer, $P_{n}$ be path, and $G=P_{n}+v_{i} v_{j}$, where $v_{i}, v_{j} \in V\left(P_{n}\right)$, with $i$ and $j$ integers $1 \leq i<j \leq n$ and, except $(i, j)=(1, n) \cdot \gamma_{\text {sleat }}(G)=3$.

Proof. Since $\Delta(G)=3, \gamma_{\text {sleat }}(G) \geq 3$. To show that $\gamma_{\text {sleat }}(G) \leq 3$, suppose $g$ is a labeling of the unicycle graph and $f$ is a SLEAT coloring of $P_{n}$ given in the proof of Theorem 2.1. We divide the proof into 4 subcases.

Case 1. $n$ is odd.

Subcase 1.1. Either $i$ or $j$ is odd. Labels vertices and edges as follows

$$
\begin{gathered}
g\left(P_{n}\right)=f\left(P_{n}\right), \\
g\left(v_{i} v_{j}\right)=2 n .
\end{gathered}
$$


Hence, we get

$$
w\left(v_{i} v_{j}\right)=g\left(v_{i}\right)+g\left(v_{i} v_{j}\right)+g\left(v_{j}\right)= \begin{cases}3 n+\frac{i+j}{2}, & \text { if } i \text { and } j \text { are odd, } \\ \frac{5}{2} n+\frac{i+j}{2}, & \text { if } i \text { or } j \text { is odd. }\end{cases}
$$

Therefore, we get a minimum value of $w\left(v_{i} v_{j}\right) \geq \frac{5 n+i+j}{2}$. Now, we will show that $w\left(v_{i} v_{j}\right)$ has different value with $w\left(v_{i} v_{i+1}\right)$. It is clear that $w\left(v_{i} v_{i+1}\right)$ has a maximum value of $\frac{5 n+1}{2}+1$. By the premise, $i+j$ has the smallest possible value attainable $i+j \geq 4$. We observe that

$$
w\left(v_{i} v_{j}\right) \geq \frac{5 n+4}{2}>\frac{5 n+1}{2}+1,
$$

which implies that $w\left(v_{i} v_{j}\right)$ is a third distinct weight.

Sub-case 1.2. $i$ and $j$ are even. Labels vertices and edges as follows

$$
\begin{gathered}
g\left(V\left(P_{n}\right)\right)=f\left(V\left(P_{n}\right)\right), \\
g\left(E\left(P_{n}\right)\right)=f\left(E\left(P_{n}\right)\right)+1, \\
g\left(v_{i} v_{j}\right)=n+1 .
\end{gathered}
$$

Since every labels of edges is increased by 1 , we will have a new weight of

$$
\begin{gathered}
w\left(v_{i} v_{j}\right)=g\left(v_{i}\right)+g\left(v_{i} v_{j}\right)+g\left(v_{j}\right), \\
w\left(v_{i} v_{i+1}\right)=\left\{\begin{aligned}
\frac{5 n+1}{2}+2 & \text { for } i \text { is even, } \\
\frac{5 n+1}{2} & \text { for } i \text { is odd. }
\end{aligned}\right.
\end{gathered}
$$

and $w\left(v_{i} v_{j}\right)=n+1+\frac{i+j}{2}$. Now, we will show that $w\left(v_{i} v_{j}\right)$ has different value with $w\left(v_{i} v_{i+1}\right)$. It is clear that $w\left(v_{i} v_{i+1}\right)$ has a minimum value of $\frac{5 n+1}{2}$. By the premise, $i+j$ has the largest possible value attainable $i+j \leq 2 n-4$. We observe that

$$
w\left(v_{i} v_{j}\right) \leq n+1+\frac{2 n-4}{2}=2 n-1<\frac{5 n+1}{2},
$$

and $w\left(v_{i} v_{j}\right)$ is a third distinct weight.

Case 2. $n$ is even.

Subcase 2.1. Either $i$ or $j$ is equal to 1 or even. Labels vertices and edges as follows

$$
\begin{gathered}
g\left(P_{n}\right)=f\left(P_{n}\right), \\
g\left(v_{i} v_{j}\right)=2 n .
\end{gathered}
$$


Hence, we get possible weights

$$
w\left(v_{i} v_{j}\right)=\left\{\begin{aligned}
3 n+\frac{i+j}{2}-2, & \text { if } i \text { and } j \text { are even, } \\
\frac{7}{2} n+\frac{j}{2}-1, & \text { if } i=1 \text { and } j \text { is even, } \\
\frac{5}{2} n+\frac{i+j}{2}-\frac{3}{2}, & \text { if } i \text { is even and } j \text { is odd, } \\
\frac{5}{2} n+\frac{i+j}{2}-\frac{3}{2}, & \text { if } i \text { is odd and } j \text { is even, } \\
3 n+\frac{j}{2}-\frac{1}{2}, & \text { if } i=1 \text { and } j \text { is odd. }
\end{aligned}\right.
$$

We will show that $w\left(v_{i} v_{j}\right)$ has different value with $w\left(v_{i} v_{i+1}\right)$. It is clear that $w\left(v_{i} v_{i+1}\right)$ has a maximum value of $\frac{5 n}{2}+1$. A minimum value for these possible weights is needed to be determined.

If both $i$ and $j$ are even, then $i+j$ has smallest value attainable $i+j \geq 2+4=6$, such that $w\left(v_{i} v_{j}\right) \geq 3 n+1$. If $i=1$ and $j$ is even, then $j$ has smallest value attainable $j \geq 4$, such that $w\left(v_{i} v_{j}\right) \geq \frac{7}{2} n+1=\frac{5}{2} n+n+1$. If $i$ is even and $j$ is odd, then $i+j$ smallest value attainable $i+j \geq 2+5=7$, such that $w\left(v_{i} v_{j}\right) \geq \frac{5}{2} n+2$. If $i=1$ and $j$ is odd, then $j$ smallest value attainable $j \geq 3$, such that $w\left(v_{i} v_{j}\right) \geq 3 n+1$.

It can be seen that $w\left(v_{i} v_{j}\right)$ has a minimum value of $\frac{5}{2} n+2 \geq \frac{5}{2} n+1$, since $n$ is even. It means $w\left(v_{i} v_{j}\right)$ is a third distinct weight.

Subcase 2.2. $i$ and $j$ are odd, and both of them does not equal to 1 . Labels as follows

$$
\begin{gathered}
g\left(V\left(P_{n}\right)\right)=f\left(V\left(P_{n}\right)\right), \\
g\left(E\left(P_{n}\right)\right)=f\left(E\left(P_{n}\right)\right)+1, \\
g\left(v_{i} v_{j}\right)=n+1 .
\end{gathered}
$$

since every labels of edges is incremented by 1 , we will have a new weight of

$$
\begin{gathered}
w\left(v_{i} v_{j}\right)=g\left(v_{i}\right)+g\left(v_{i} v_{j}\right)+g\left(v_{j}\right), \\
w\left(v_{i} v_{i+1}\right)=\left\{\begin{aligned}
\frac{5 n}{2}, & \text { if } i \text { is even, } \\
\frac{5 n}{2}+2, & \text { if } i \text { is odd. }
\end{aligned}\right.
\end{gathered}
$$

and $w\left(v_{i} v_{j}\right)=n+\frac{i+j}{2}$. We will show that this $w\left(v_{i} v_{j}\right)$ has different value with $w\left(v_{i} v_{i+1}\right)$. It is clear that $w\left(v_{i} v_{i+1}\right)$ has a minimum value of $\frac{5 n}{2}$. By the premise, $i+j$ has the largest attainable value $i+j \leq 2 n-4$. We observe that $w\left(v_{i} v_{j}\right) \leq n+\frac{2 n-4}{2}=2 n-2<\frac{5 n}{2}$. Therefore, $w\left(v_{i} v_{j}\right)$ is a third distinct weight.

Since in every case $\gamma_{\text {sleat }}(G) \leq 3$, we can deduce that $g$ is SLEAT of $G$ with $\gamma_{\text {sleat }}(G)=3$.

\subsubsection{Path with some edges addition}

This method of edge addition can be done multiple times if it meets certain premises. We need to introduce a convenient even function, defined as follows

$$
\operatorname{even}(n)= \begin{cases}1, & \text { if } n \text { is even } \\ 0, & \text { if } n \text { is odd }\end{cases}
$$



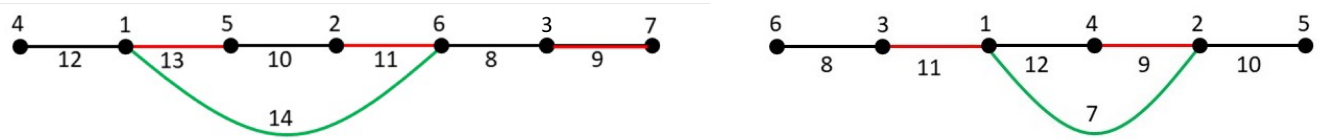

Figure 2: SLEAT coloring of $G=P_{7}+v_{2} v_{5}$ and $G=P_{6}+v_{3} v_{5}$

Theorem 2.3. Let $n \geq 4$ be integer, $P_{n}$ be path, and $G=P_{n}+\left\{v_{i-k} v_{j-k} \mid 0 \leq k \leq p, 0 \leq p \leq\right.$ $\min \{i-1+\operatorname{even}(n), j-i-1\}\}$ where $v_{i-k}, v_{j-k} \in V\left(P_{n}\right), i$ and $j$ satisfy $1 \leq i<j \leq n, i+j$ is odd, and $(i, j) \neq(1, n) \cdot \gamma_{\text {sleat }}(G)=3$

Proof. Since $\Delta(G)=3, \gamma_{\text {sleat }}(G) \geq 3$. To show that $\gamma_{\text {sleat }}(G) \leq 3$, suppose $g$ is a labeling of $G$ and $f$ is a SLEAT coloring of $P_{n}$ given in the proof of Theorem 2.1.

Labels vertices and edges as follows

$$
\begin{gathered}
g\left(P_{n}\right)=f\left(P_{n}\right), \\
g\left(v_{i-k} v_{j-k}\right)=2 n+k .
\end{gathered}
$$

The weight of edge are the following

$w\left(v_{i-k} v_{j-k}\right)=g\left(v_{i-k}\right)+g\left(v_{i-k} v_{j-k}\right)+g\left(v_{j-k}\right)=\left\{\begin{aligned} \frac{5}{2} n+\frac{i+j}{2}, & \text { if } n \text { is odd, } i+j \text { is odd, } \\ \frac{5}{2} n+\frac{i+j}{2}-\frac{3}{2}, & \text { if } n \text { is even, } i+j \text { is odd. }\end{aligned}\right.$

We will show that $w\left(v_{i-k} v_{j-k}\right)$ has different value with $w\left(v_{i} v_{i+1}\right)$. It is clear that $w\left(v_{i} v_{i+1}\right)$ has a maximum value of $\frac{5 n+1}{2}+1$. A minimum value for these possible weights is needed to be determined.

If both $n$ and $i+j$ are odd, then smallest value attainable $i+j \geq 1+3=4$, such that $w\left(v_{i-k} v_{j-k}\right) \geq \frac{5}{2} n+2$. If $n$ is even, $i \neq 1$, and $i+j$ is odd, then smallest value attainable $i+j \geq 2+5=7$, such that $w\left(v_{i-k} v_{j-k}\right) \geq \frac{5}{2} n+2$.

Since $n \geq 4, w\left(v_{i-k} v_{j-k}\right)$ has a minimum value of $\frac{5}{2} n+2>\frac{5 n+1}{2}+1$. As a result, $w\left(v_{i-k} v_{j-k}\right)$ is a third distinct weights. Therefore, $g$ is a SLEAT of $G$ with $\gamma_{\text {sleat }}(G)=3$.

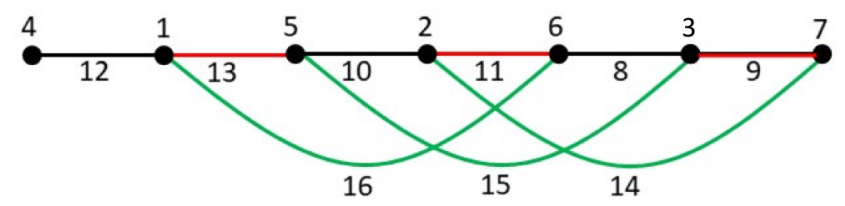

Figure 3: SLEAT coloring of $G=P_{7}+\left\{v_{4-k} v_{7-k} \mid 0 \leq k \leq 2\right\}$ 


\subsection{Connecting disjoint paths}

Another kind of graph that is observed in this study is some disjoint paths with addition of edge(s) that connects some paths. Two kinds of this family of graph are hedge graph and hedgerow graph. These two graph will be defined in the proceeding subsections.

\subsubsection{Hedge graph}

To start, we observe two disjoint paths with addition of one edge that connects the paths. Such graph is called hedge graph $H d$. Before giving the formal definition of hedge graph, we want to exclude the possibility of connection that forms a simple path. For arbitrary set $A, B$, we use the cartesian products of the two sets $A \times B=\{(a, b) \mid a \in A, b \in B\}$ in order to have simple notations. Let $P_{m}$ and $P_{n}$ be path with $V\left(P_{m}\right)=\left\{v_{k} \mid 1 \leq k \leq m\right\}$ and $V\left(P_{n}\right)=\left\{u_{l} \mid 1 \leq l \leq n\right\}$. We define $H d(m, n, i, j)$ as follows

$$
\begin{gathered}
V(H d(m, n, i, j))=V\left(P_{m}\right) \cup V\left(P_{n}\right), \\
E(H d(m, n, i, j))=E\left(P_{m}\right) \cup E\left(P_{n}\right) \cup\left\{v_{i} u_{j} \mid 1 \leq i \leq m, 1 \leq j \leq n,(i, j) \notin(1, n) \times(1, m)\right\} .
\end{gathered}
$$

Theorem 2.4. For integers $n \geq m \geq 3$, if $G=H d(m, n, i, j)$, then $\gamma_{\text {sleat }}(G)=3$.

Proof. Since $\Delta(G)=3, \gamma_{\text {sleat }}(G) \geq 3$. To show that $\gamma_{\text {sleat }}(G) \leq 3$, suppose $g$ is a labeling of $G$ and $f$ is a SLEAT coloring of $P_{n}$ given in the proof of Theorem 2.1.

Case 1. $i \neq j$.

Add an edge $v_{1} u_{1}$ to form a $P_{m+n}$, apply labeling $f$ in a path $P_{m+n}$ from $v_{n}$ to $u_{m}$, then remove $v_{1} u_{1}$. Now, $g$ is a labelings that follows

$$
\begin{gathered}
g\left(v_{i} u_{j}\right)=f\left(v_{1} u_{1}\right), \\
g(V(G))=f\left(V\left(P_{m+n}\right)\right), \\
g\left(E(G) \backslash\left\{v_{i} u_{j}\right\}\right)=f\left(E\left(P_{m+n}\right) \backslash\left\{v_{1} u_{1}\right\}\right) .
\end{gathered}
$$

If $n+m$ is odd, we have the following weight

$$
w\left(v_{i} v_{i+1}\right)=w\left(u_{i} u_{i+1}\right)= \begin{cases}\frac{5 n+1}{2}+1, & \text { if } i \text { is even } \\ \frac{5 n+1}{2}-1, & \text { if } i \text { is odd. }\end{cases}
$$

As for $n+m$ is even, we have the following weight

$$
w\left(v_{i} v_{i+1}\right)=w\left(u_{i} u_{i+1}\right)= \begin{cases}\frac{5 n}{2}-1, & \text { if } i \text { is even } \\ \frac{5 n}{2}+1, & \text { if } i \text { is odd }\end{cases}
$$

We get $g\left(v_{1}\right)+g\left(v_{i} u_{j}\right)+g\left(u_{1}\right)=w\left(v_{i} v_{i+1}\right)=w\left(u_{i} u_{i+1}\right)$ for even $i$. Now, we can observe the partition $\left\{g\left(v_{i}\right)\right\}$ and $\left\{g\left(u_{j}\right)\right\}$ for arbitrary $i$ and $j$, to find that there is no pair of $\left(g\left(v_{i}\right), g\left(u_{j}\right)\right)$ such that $g\left(v_{i}\right)+g\left(u_{j}\right)=g\left(v_{1}\right)+g\left(u_{1}\right)$ which satisfies $i \neq j$. Hence, $w\left(v_{i} u_{j}\right) \neq w\left(v_{i} v_{i+1}\right)$ and $w\left(v_{i} u_{j}\right) \neq w\left(v_{i} v_{i+1}\right)$ for even $i$. 
Next, we get $g\left(v_{1}\right)+g\left(v_{i} u_{j}\right)+g\left(u_{1}\right)-2=w\left(v_{i} v_{i+1}\right)=w\left(u_{i} u_{i+1}\right)$ or $g\left(v_{1}\right)+g\left(v_{i} u_{j}\right)+$ $g\left(u_{1}\right)+2=w\left(v_{i} v_{i+1}\right)=w\left(u_{i} u_{i+1}\right)$ for odd $i$. Again, we can observe the partition $\left\{g\left(v_{i}\right)\right\}$ and $\left\{g\left(u_{j}\right)\right\}$ for arbitrary $i$ and $j$, to find that there is no pair of $\left(g\left(v_{i}\right), g\left(u_{j}\right)\right)$ such that $g\left(v_{i}\right)+g\left(u_{j}\right)=$ $g\left(v_{1}\right)+g\left(u_{1}\right)-2$ or $g\left(v_{i}\right)+g\left(u_{j}\right)=g\left(v_{1}\right)+g\left(u_{1}\right)+2$. Hence, $w\left(v_{i} u_{j}\right) \neq w\left(v_{i} v_{i+1}\right)$ and $w\left(v_{i} u_{j}\right) \neq w\left(v_{i} v_{i+1}\right)$ for odd $i$.

Case 2. $i=j$, and $n \neq m$.

Add an edge $v_{n} u_{m}$ to form a $P_{m+n}$, apply labeling $f$ to path $P_{m+n}$ from $v_{1}$ to $u_{1}$, then remove $v_{n} u_{m}$. Now, $g$ is a labelings that follows

$$
\begin{gathered}
g\left(v_{i} u_{j}\right)=f\left(v_{n} u_{m}\right), \\
g(V(G))=f\left(V\left(P_{m+n}\right)\right), \\
g\left(E(G) \backslash\left\{v_{i} u_{j}\right\}\right)=f\left(E\left(P_{m+n}\right) \backslash\left\{v_{n} u_{m}\right\}\right) .
\end{gathered}
$$

If $n+m$ is odd, we have the following weight

$$
w\left(v_{i} v_{i+1}\right)=w\left(u_{i+1} u_{i+2}\right)= \begin{cases}\frac{5 n+1}{2}+1, & \text { if } i \text { is even, } \\ \frac{5 n+1}{2}-1, & \text { if } i \text { is odd. }\end{cases}
$$

With the addition of $w\left(u_{1} u_{2}\right)=\frac{5 n+1}{2}+1$. As for $n+m$ is even, we have the following weight

$$
w\left(v_{i} v_{i+1}\right)=w\left(u_{i} u_{i+1}\right)= \begin{cases}\frac{5 n}{2}-1, & \text { if } i \text { is even, } \\ \frac{5 n}{2}+1, & \text { if } i \text { is odd. }\end{cases}
$$

We get $g\left(v_{n}\right)+g\left(v_{i} u_{j}\right)+g\left(u_{m}\right)=w\left(v_{i} v_{i+1}\right)=w\left(u_{i+1} u_{i+2}\right)=w\left(u_{1} u_{2}\right)$ for $n$ and $i$ that have the same parity. Now, we can observe the partition $\left\{g\left(v_{i}\right)\right\}$ and $\left\{g\left(u_{i}\right)\right\}$ for arbitrary $i$, to find that there is no pair of $\left(g\left(v_{i}\right), g\left(u_{i}\right)\right)$ such that $g\left(v_{i}\right)+g\left(u_{i}\right)=g\left(v_{n}\right)+g\left(u_{m}\right)$. Hence, $w\left(v_{i} u_{j}\right)$ does not equal any of $w\left(v_{i} v_{i+1}\right), w\left(u_{i+1} u_{i+2}\right)$, or $w\left(u_{1} u_{2}\right)$.

Next, we get $g\left(v_{n}\right)+g\left(v_{i} u_{j}\right)+g\left(u_{m}\right)-2$ or $g\left(v_{1}\right)+g\left(v_{i} u_{j}\right)+g\left(u_{1}\right)+2$ to be equal with $w\left(v_{i+1} v_{i+2}\right)=w\left(u_{i} u_{i+1}\right)=w\left(v_{1} v_{2}\right)$ for $n$ and $i$ have different parity. Again, we can observe the partition $\left\{g\left(v_{i}\right)\right\}$ and $\left\{g\left(u_{i}\right)\right\}$ for arbitrary $i$, to find that there is no pair of $\left(g\left(v_{i}\right), g\left(u_{i}\right)\right)$ such that $g\left(v_{i}\right)+g\left(u_{i}\right)=g\left(v_{n}\right)+g\left(u_{m}\right)-2$ or $g\left(v_{i}\right)+g\left(u_{i}\right)=g\left(v_{n}\right)+g\left(u_{m}\right)+2$. Hence, $w\left(v_{i} u_{j}\right)$ does not equal any of $w\left(v_{i+1} v_{i+2}\right), w\left(u_{i} u_{i+1}\right)$, or $w\left(v_{1} v_{2}\right)$.

Case 3. $i=j$, and $n=m$, except $n=3$.

Since the graph with $i=2$ is isomorphic with $i=n-1$, it is only necessary to determine labels for $i \geq 3$.

Add an edge $v_{1} u_{1}$ to form a path $P_{m+n}$, apply labeling $f$ to $P_{m+n}$ from $v_{n}$ to $u_{m}$, then remove $v_{1} u_{1}$.

If $n$ is odd, $g$ is a labelings that follows

$$
g\left(v_{i} u_{j}\right)=f\left(u_{1} u_{2}\right)
$$




$$
\begin{gathered}
g\left(u_{1} u_{2}\right)=f\left(v_{1} u_{1}\right), \\
g(V(G))=f\left(V\left(P_{m+n}\right)\right), \\
g\left(E(G) \backslash\left\{v_{i} u_{j} \cup u_{1} u_{2}\right\}\right)=f\left(E\left(P_{m+n}\right) \backslash\left\{v_{1} u_{1} \cup u_{1} u_{2}\right\}\right) .
\end{gathered}
$$

If $n$ is even, $g$ is a labelings that follows

$$
\begin{gathered}
g\left(v_{i} u_{j}\right)=f\left(v_{1} v_{2}\right), \\
g\left(v_{1} v_{2}\right)=f\left(v_{1} u_{1}\right), \\
g(V(G))=f\left(V\left(P_{m+n}\right)\right), \\
g\left(E(G) \backslash\left\{v_{i} u_{j} \cup v_{1} v_{2}\right\}\right)=f\left(E\left(P_{m+n}\right) \backslash\left\{v_{1} u_{1} \cup v_{1} v_{2}\right\}\right) .
\end{gathered}
$$

By preceding labelings, we have the following weight

$$
w\left(v_{i+1} v_{i+2}\right)=w\left(u_{i+1} u_{i+2}\right)= \begin{cases}\frac{5 n}{2}-1, & \text { if } i \text { is even, } \\ \frac{5 n}{2}+1, & \text { if } i \text { is odd. }\end{cases}
$$

In addition, if $n$ is odd, $w\left(v_{1} v_{2}\right)=w\left(v_{i+1} v_{i+2}\right)$ and $w\left(u_{1} u_{2}\right)=w\left(v_{i} u_{j}\right)$. While if $n$ is even, $w\left(u_{1} u_{2}\right)=w\left(u_{i+1} u_{i+2}\right)$ and $w\left(v_{1} v_{2}\right)=w\left(v_{i} u_{j}\right)$. By case 1 , it is already proven that $w\left(v_{i} u_{j}\right)$ is distinct.

Case 4. $i=j=2$, and $n=m=3$.

This is the only one specific graph that does not belong to any preceding case. Thus, we can simply do an enumeration for completing the theorem. Labels the graph $G$ as in Figure 4.

Since every possible graph is covered, therefore $\gamma_{\text {sleat }}(H d(m, n, i, j))=3$.
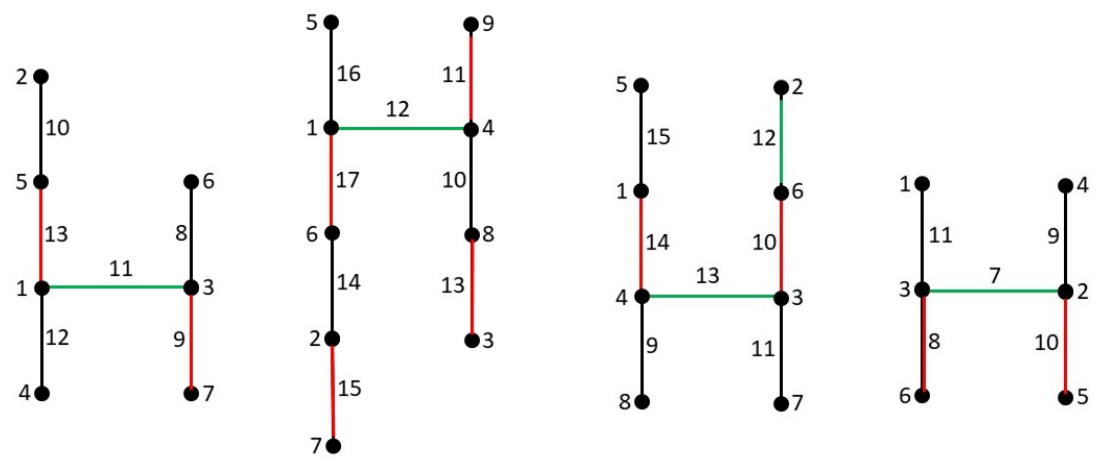

Figure 4: SLEAT coloring of $H d(4,3,3,2), H d(5,4,2,2), H d(4,4,3,3)$, and $H d(3,3,2,2)$ 


\subsubsection{Hedgerow}

Suppose we have $m$ disjoint paths with length of $n$. Every neighbouring path is connected with an edge that is uniform through every two connected paths. Such graph is called hedgerow graph $H r$. Let $m P_{n}$ be the disjoint union of $m$ number of $P_{n}$ with $V\left(m P_{n}\right)=\left\{v_{l}^{k} \mid 1 \leq l \leq n, 1 \leq k \leq\right.$ $m\}$. Formally, we define hedgerow graph $\operatorname{Hr}(m, n, i, j)$ as follows

$$
\begin{gathered}
V(H r(m, n, i, j))=V\left(m P_{n}\right), \\
E(H r(m, n, i, j))=E\left(m P_{n}\right) \cup\left\{v_{i}^{k} v_{j}^{k+1} \mid 1 \leq i \leq n, 1 \leq j \leq n, 1 \leq k \leq m-1\right\} .
\end{gathered}
$$

We start an attempt to find hedgerow for $m \geq 3$. We only find the value of $\gamma_{\text {sleat }}$ for hedgerow graph for some $(m, n, i, j)$.

Theorem 2.5. Let integer $m \geq 2$ and integer $n \geq 2$. For $G \cong \operatorname{Hr}(m, n, i, j)$,

1. If $n$ is even, $i \neq j$, and $i \neq n-j+1$, then $\gamma_{\text {sleat }}(G)=3$.

2. If $m$ is odd, $i=j$, and $i \notin\{1, n\}$, then $\gamma_{\text {sleat }}(G)=4$.

Proof. By Theorem 2.1, we have $f$, SLEAT labelings of path graph. Suppose $g$ is SLEAT labelings of the connected multiple path.

Case 1. $n$ is even, $i \neq j$, and $i \neq n-j+1$

Since $\Delta(G)=3, \gamma_{\text {sleat }}(G) \geq 3$. Next, we will show that $\gamma_{\text {sleat }}(G) \leq 3$. Since the graph has reflexive form, if $i=1$ we can redefine $(i, j)=\left(1, j^{\prime}\right)$ into $(i, j)=\left(j^{\prime}, 1\right)$, thus we only consider $i \geq 2$. Add edges $v_{n}^{k} v_{1}^{k+1}$ for every integer $k \in[1, m]$, forming $P_{m n}$, apply $f$ in a path from $v_{1}^{1}$ to $v_{n}^{m}$. Now, for every integer $k \in[1, m-1], g$ is a labeling that follows

$$
\begin{gathered}
g\left(v_{i}^{k} v_{j}^{k+1}\right)=f\left(v_{n}^{k} v_{1}^{k+1}\right), \\
g\left(V(G)=f\left(V\left(P_{m n}\right)\right),\right. \\
g\left(E \left(G \backslash\left(\cup_{k}\left\{v_{i}^{k} v_{j}^{k+1}\right\}\right)=f\left(E \left(P_{m n} \backslash\left(\cup_{k}\left\{v_{n}^{k} v_{1}^{k+1}\right\}\right) .\right.\right.\right.\right.
\end{gathered}
$$

In the proof of Theorem 2.4, we have shown that $w\left(v_{i}^{1} v_{j}^{2}\right)$ with two other weights in the paths, with restriction of $i \neq n-j+1$. Hence, we have the weights

$$
w\left(v_{i}^{k} v_{i+1}^{k}\right)= \begin{cases}\frac{5}{2} n m-1, & \text { if } i \text { is even, } \\ \frac{5}{2} n m+1, & \text { if } i \text { is odd. }\end{cases}
$$

Originally, $f\left(v_{n}^{k}\right)+f\left(v_{n}^{k} v_{1}^{k+1}\right)+f\left(v_{1}^{k+1}\right)$ is constant for every integer $k \in[1, m-1]$. Since $g\left(v_{n}^{k}\right)-g\left(v_{i}^{k}\right)$ and $g\left(v_{1}^{k+1}\right)-g\left(v_{j}^{k+1}\right)$ are constant for the same $k$, we have third distinct weight $w\left(v_{i}^{k} v_{j}^{k+1}\right)$. Hence, $\gamma_{\text {sleat }}(G)=3$.

Case 2. $m$ is odd, $i=j$, and $i \notin\{1, n\}$ 
Since $\Delta(G)=4, \gamma_{\text {sleat }}(G) \geq 4$. Next, we will show that $\gamma_{\text {sleat }}(G) \leq 4$. Add edges $v_{n}^{k} v_{n}^{k+1}$ for every odd integer $k \in[1, m-2]$ and $v_{1}^{k} v_{1}^{k+1}$ for every even integer $k \in[2, m-1]$, forming $P_{m n}$, apply $f$ in a path from $v_{1}^{1}$ to $v_{n}^{m}$. Now, for every integer $k \in[1, m-1]$ and integer $k^{\prime} \in\left[1, \frac{m-1}{2}\right], g$ is a labeling that follows

$$
\begin{gathered}
g\left(v_{i}^{k} v_{i}^{k+1}\right)=\left\{\begin{array}{l}
f\left(v_{n}^{k} v_{n}^{k+1}\right) \text { for } k \text { is odd, } \\
f\left(v_{1}^{k} v_{1}^{k+1}\right) \text { for } k \text { is even, }
\end{array}\right. \\
g(V(G))=f\left(V\left(P_{m n}\right)\right), \\
g\left(E\left(G \backslash\left(\cup_{k}\left\{v_{i}^{k} v_{i}^{k^{\prime}+1}\right\}\right)\right)\right)=g\left(E\left(P_{m n} \backslash\left(\cup_{k}^{\prime}\left\{v_{1}^{2 k^{\prime}} v_{1}^{2 k^{\prime}+1}, v_{n}^{2 k^{\prime}-1} v_{n}^{2 k^{\prime}}\right\}\right)\right)\right) .
\end{gathered}
$$

Since $f\left(v_{1}^{2 k^{\prime}} v_{1}^{2 k^{\prime}+1}\right)-f\left(v_{n}^{2 k^{\prime}-1} v_{n}^{2 k^{\prime}}\right)$ is constant for every $k^{\prime} \in\left[1, \frac{m-1}{2}\right], w\left(v_{1}^{2 k^{\prime}} v_{1}^{2 k^{\prime}+1}\right)$ is constant for every $k, w\left(v_{n}^{2 k^{\prime}-1} v_{n}^{2 k^{\prime}}\right)$ is constant for every $k$, and $w\left(v_{1}^{2 k^{\prime}} v_{1}^{2 k^{\prime}+1}\right) \neq w\left(v_{n}^{2 k^{\prime}-1} v_{n}^{2 k^{\prime}}\right)$. Hence, $\gamma_{\text {sleat }}(G)=4$.

Therefore, the theorem holds.
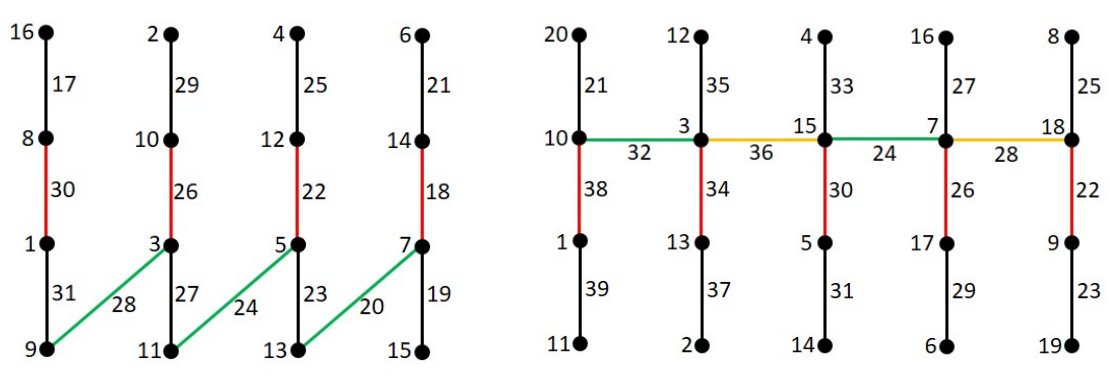

Figure 5: SLEAT coloring of $\operatorname{Hr}(4,4,4,3)$ and $\operatorname{Hr}(5,4,2,2)$

The same procedure can also be applied if $i \in\{1, n\}$ for $G$, but it will not give an exact value of $\gamma_{\text {sleat }}(G)$ since $\gamma(G)$ is still lower than the number of the weights. Despite the uncertainty, we establish the corollary as follows.

Corollary 2.1. Let integer $n \geq 2$ and odd integer $m \geq 3$. If $G \cong \operatorname{Hr}(m, n, i, i)$, then $3 \geq$ $\gamma_{\text {sleat }}(G) \geq 4$

It can be seen that the graph from the corollary is isomorphic with the comb product of $P_{m}$ with $P_{n-1}$. We revised a theorem that given by Kurniawati et al. in [7].

We attempted to find the value for some $(m, n, i, j)$ that did not satisfy preceding theorem premises, but it is not completed yet. It is left as an open problem.

Open Problem 1. Find the value of $\gamma_{\text {sleat }}(\operatorname{Hr}(m, n, i, j))$ for remaining cases of $(m, n, i, j)$. 


\subsection{Amalgamation of Star}

We proceed by finding SLEAT for star graph. Star graph is denoted as $S_{m}$ where $V\left(S_{m}\right)=$ $\left\{c, v_{i} \mid 1 \leq i \leq m\right\}$ and $E\left(S_{m}\right)=\left\{c v_{i} \mid 1 \leq i \leq m\right\}$. Since every edge in the graph is adjacent, $\gamma\left(S_{m}\right)=m$. Since $\gamma(G) \leq \gamma_{\text {sleat }}(G) \leq|E(G)|$ for arbitrary graph $G$, it is clear that $\gamma_{\text {sleat }}\left(S_{m}\right)=$ $m$. If one want an example how to achieve such graph, labels as follows $f\left(v_{i}\right)=i, f(c)=$ $m+1, f\left(c v_{i}\right)=m+i+1$. The weights is going to be consecutive by 2 as the common difference.

In this section, we study an amalgamation of star and graph which have certain $\gamma_{\text {sleat }}$.

Theorem 2.6. Suppose $G_{1}$ be a SLEAT colorable graph and $G_{2} \cong \operatorname{Amal}\left(S_{m}, G_{1} ; c\right)$ which vertex c is a center in $S_{m}$, for integer $m \geq 1$. $\gamma_{\text {sleat }}\left(G_{2}\right) \leq \gamma_{\text {sleat }}\left(G_{1}\right)+m$

Proof. Let $n_{v}=\left|V\left(G_{1}\right)\right|$ and $n_{e}=\left|E\left(G_{1}\right)\right|$. Suppose $g$ is the SLEAT labelings of $G_{1}$ and $f$ is the SLEAT labelings of $G_{2}$. Labels as follows:

$$
\begin{gathered}
g\left(V\left(G_{1}\right)\right)=f\left(V\left(G_{1}\right)\right) \\
g\left(E\left(G_{1}\right)\right)=f\left(E\left(G_{1}\right)\right)+m \\
g\left(v_{i}\right)=n_{v}+i \\
g\left(c v_{i}\right)=n_{v}+n_{e}+m+i .
\end{gathered}
$$

Let $w_{f}$ is weights as a result of $f$. Now, we define $w_{f}^{\prime}=w_{f}+m$. Next, we define $w_{s}$ as $w_{s}=g\left(v_{i}\right)+g\left(c v_{i}\right)+g(c)=2 n_{v}+n_{e}+m+2 i+f(c)$. Now, every distinct $w_{f}^{\prime}$ and every distinct $w_{s}$ are weights that is generated from $g$. What is left to prove is $w_{f}^{\prime} \neq w_{s}$ for arbitrary possible weights.

Let $p$ is the lowest $f(c)$ for arbitrary $c$. Since $p$ is adjacent with any $w_{f}^{\prime}$, we can observe the upper bound for $w_{f}^{\prime}$ by assuming $w_{f}^{\prime}$ contains the largest vertex label $n_{v}$, and largest edge label $n_{v}+n_{e}+m$. As a result,

$$
w_{f}^{\prime} \leq n_{v}+n_{v}+n_{e}+m+p=2 n_{v}+n_{e}+m+p
$$

Now, we can find the lower bound any $w_{s}$, by choosing the lowest possible $i=1$, then

$$
w_{s} \geq 2 n_{v}+n_{e}+m+p+2
$$

It is clear that $2 n_{v}+n_{e}+m+p+2 \geq 2 n_{v}+n_{e}+m+p$. Therefore, $w_{f}^{\prime} \neq w_{s}$ for arbitrary possible weights. This holds the theorem.

The preceding theorem applies for any vertex $c$ in graph $G_{1}$. By choosing certain $c$, we have Corollary 2.2.

Corollary 2.2. Let $G_{1}$ is a SLEAT colorable graph and $G_{2} \cong \operatorname{Amal}\left(S_{m}, G_{1} ; c\right)$ which vertex $c$ is a center in $S_{m}$, for integer $m \geq 1$. If $\operatorname{deg}(c)=\Delta\left(G_{2}\right), \gamma_{\text {sleat }}\left(G_{2}\right)=\gamma_{\text {sleat }}\left(G_{1}\right)+m$. 

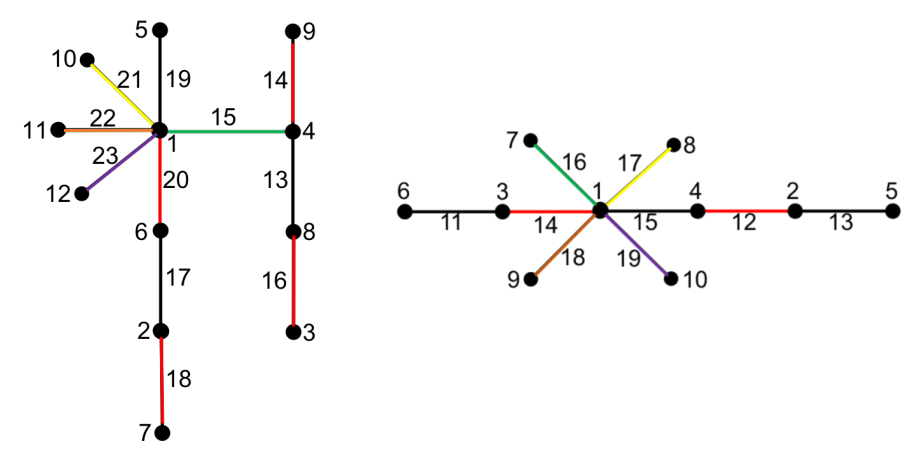

Figure 6: SLEAT coloring of $\operatorname{Amal}\left(S_{3}, \operatorname{Hd}(5,4,2,2), c\right)$ and $\operatorname{Amal}\left(S_{4}, P_{6}, c\right)$

\section{Summary}

Here we summarize our results in Table 1.

Table 1: Summary

\begin{tabular}{|c|c|c|c|}
\hline Graph & Notation & $\gamma_{\text {sleat }}$ & Condition \\
\hline Path & $P_{n}$ & 2 & \\
\hline Path + edge & $P_{n}+v_{i} v_{j}$ & 3 & $\begin{array}{l}1 \leq i<j \leq n, \text { and }(i, j) \neq \\
(1, n)\end{array}$ \\
\hline $\begin{array}{l}\text { Path + multiple } \\
\text { edges }\end{array}$ & $P_{n}+\left\{v_{i-k} v_{j-k} \mid 0 \leq k \leq p\right\}$ & 3 & $\begin{array}{l}1 \leq i<j \leq n, i+j \text { is odd, } \\
0 \leq p \leq \min \{i-1+\operatorname{even}(n), j- \\
i-1\} \text { and }(i, j) \neq(1, n)\end{array}$ \\
\hline Hedge graph & $H d(m, n, i, j)$ & 3 & $\begin{array}{l}v_{i} \in V\left(P_{n}\right), u_{j} \in V\left(P_{m}\right), \text { and } \\
(i, j) \notin(1, n) \times(1, m)\end{array}$ \\
\hline $\begin{array}{l}\text { Hedgerow } \\
\text { graph }\end{array}$ & $H r(m, n, i, j)$ & 3 & $n$ is even, $i \neq j$, and $i \neq n-j+1$ \\
\hline $\begin{array}{l}\text { Hedgerow } \\
\text { graph }\end{array}$ & $\operatorname{Hr}(m, n, i, j)$ & 4 & $m$ is odd, $i=j$, and $i \notin\{1, n\}$ \\
\hline Star & $S_{n}$ & $n$ & \\
\hline $\begin{array}{l}\text { Amalgamation } \\
\text { of star }\end{array}$ & $\operatorname{Amal}\left(G, S_{n} ; c\right)$ & $\begin{array}{lr}\text { at } & \text { most } \\
\gamma_{\text {sleat }}(G)+n\end{array}$ & $\begin{array}{l}c \in V(G), V\left(S_{n}\right) \text { and } c \text { is center } \\
\text { in } S_{n}\end{array}$ \\
\hline $\begin{array}{l}\text { Amalgamation } \\
\text { of star }\end{array}$ & $\operatorname{Amal}\left(G, S_{n} ; c\right)$ & $\gamma_{\text {sleat }}(G)+n$ & $\begin{array}{l}c \in V(G), V\left(S_{n}\right), c \text { is center in } \\
S_{n}, \text { and } \operatorname{deg}(c)=\Delta(G)\end{array}$ \\
\hline
\end{tabular}

\section{Acknowledgement}

Part of this research is funded by Universitas Indonesia through Hibah PITTA B UI under contract 168 No. NKB-0622/UN2.R3.1/HKP.05.00/2019. 


\section{References}

[1] I. H. Agustin, Dafik, M. Hasan, R. Alfarisi, and R. M. Prihandini, On the local edge antimagic coloring of graphs, Far East J. Math. Sci. 102 (9) (2017) 1925-1941.

[2] I. H. Agustin, R. Alfarisi, Dafik, and A. I. Kristiana, On super local antimagic total edge coloring of some wheel related graphs, AIP Conf. Proc. 2014 (2018) 020088.

[3] S. Arumugam, K. Premalatha, M. Bača, and A. Semaničová-Feňovčíková, Local antimagic vertex coloring of a graph, Graphs Combin. 33 (2017) 275-285.

[4] R. Bodendiek and G. Walther, On arithmetic antimagic edge labelings of graphs, Mitt. Math. Ges. Hamburg 17 (1998) 85-99.

[5] J. A. Gallian, A dynamic survey of graph labeling, Electron. J. Combin. 21 (2018)\#DS6.

[6] N. Hartsfield and G. Ringel, Pearls in Graph Theory, Academic Press, San Diego (1990).

[7] E. Y. Kurniawati, I. H. Agustin, Dafik, and R. Alfarisi, Super local edge antimagic total coloring of $P_{n} \triangleright$ H, IOP Conf. Series: J. Phys.: Conf. Series 1008 (2018) 012036 1-11.

[8] E. Y. Kurniawati, I. H. Agustin, Dafik, and Marsidi, On the vertex local antimagic total labeling chromatic number of $G \odot K_{2}$, IOP Conf. Series: J. Phys.: Conf. Series 1211 (2019) $0120181-8$. 